\title{
Enhancing Immersiveness in AR-Based Product Design*
}

\author{
Taejin $\mathrm{Ha}^{1}$, Yeongmi Kim² ${ }^{2}$ Jeha Ryu ${ }^{2}$, and Woontack Woo ${ }^{1}$ \\ ${ }^{1}$ GIST U-VR Lab, \\ ${ }^{2}$ GIST HMCI Lab, \\ Gwangju, 500-712, Korea \\ \{tha, kym, ryu, wwoo\}@gist.ac.kr
}

\begin{abstract}
Recently, various AR-based product design methodologies have been introduced. In this paper, we propose technologies for enhancing the immersive realization of virtual objects, where, by adapting tangible objects we can provide touch sensation to users. A 3D model of the same scale overlays the whole area of the tangible object so the marker area is invisible. This contributes to enhancing immersion. Also, the hand occlusion problem when the virtual objects overlay the user's hands is partially solved, providing more immersive and natural images to users. Finally, multimodal feedback also creates better immersion. In our work, both vibrotactile feedback through page motors and sound feedback are considered. In our scenario, a game-phone model is selected, by way of proposed augmented vibrotactile feedback, hands occlusion-reduced visual effects and sound feedback are provided to users. These proposed methodologies will contribute to a better immersive realization of the conventional AR system.
\end{abstract}

Keywords: AR-based Product Design, Hands Occlusion, Tangible Object, Interaction using Hands, Vibrotactile.

\section{Introduction}

In general, when customers buy an electronic product, they consider several criteria, such as price, maker, durability, operating time, design etc. Among these, contemporary product design has become an increasingly important consideration for prospective customers. As a response to these consumer demands, product design cycles are getting shorter. Therefore rapid product design methodologies are necessary to keep up with these trends. Generally mock-up models in product design are based on 3D models in CAD. This enables customers to experience the product before production, and designers can obtain direct feedback on colours or position of buttons from customers. However, as the product design cycle becomes shorter, the process related to the mock-up model design and its reproduction should also be significantly reduced.

In the conventional desktop-based product design environment, users inspect virtual objects through a monitor in a fixed position, using a mouse or a keyboard to

\footnotetext{
* This research is supported by the UCN Project, the MIC 21C Frontier R\&D Program in Korea, ICRC and RBRC at GIST. 
model virtual objects. In that situation, view space is in a monitor and interaction space is constructed through a mouse or a keyboard on the table. This can cause unnatural interaction because each space is not collocated. To compensate for the above problem, AR-based design methodologies have been introduced [1-3]. In this case, the user is usually wearing an HMD and can interact with virtual objects in a real environment. This enables collocation between view space and interaction space, and therefore a more intuitive interaction is possible.

However, several problems exist in applying AR technology to product design. First of all, usually an augmented virtual object has no physical entity so we can not touch the virtual object, and this reduces immersion. Phantom devices [4] can be one method for touching virtual objects but this requires fixed positions and users can only touch objects through the end effecter of a pen. Second, an augmented virtual object can occlude users' hands because virtual objects just overlay the whole marker area (e.g. the tangible object). This may cause visually awkward images and it also can reduce immersion or naturalness. Also hand segmentation is difficult under changing lighting conditions. Lastly, in terms of interaction, conventional AR applications mainly focus on visual feedback. The most augmented objects do not provide any tactile feedback even though providing tactile cues is helpful for generating their presence. Touch sensations and auditory sensations are also necessary for better immersion.

In our proposed AR-based product design, users can interact with virtual objects which are overlaid on tangible objects. By registering the nonphysical virtual object to the physical tangible object on a corresponding scale, better immersion can be achieved. The virtual object can have a physical entity, thus reducing the gap between virtual environments and real environments. Second, in order to reduce the hand occlusion phenomenon under changing lighting conditions, our method extracts convexes of tangible objects, and then estimate an unobservable underlying probability density function only in that convex area through the "Kernel Density Estimation" [5]. The peaks and valley of the histogram are then calculated to establish the thresholds for segmenting the hand object. Lastly, to provide multimodal feedback to users, visual, sound and vibration feedback is provided through a vibration module embedded in the tangible object which reacts when events occur.

In application, we implemented the immersive modeling test bed for the functionality test of a game-phone. Users are able to control cars in racing game by grasping and tilting a tangible object If the car collides with a wall then vibration and sound feedback are provided for better immersion, enabling multimodal feedback. The proposed methodologies in this paper will contribute to improvements in conventional AR-based product design.

Section 2 provides the detailed algorithm, and section 3 addresses implementation and shows the experiment results of our proposed method. Finally we present conclusions and future work in section 4.

\section{Enhancing Immersiveness in AR-Based Product Design}

Users wearing a 3D HMD on their head interact with virtual objects by grasping a tangible object. This tangible object is a mock-up model of a game-phone. Multiple fiducial markers are attached to provide 3D information. A 3D model is overlaid on 
the whole area of the tangible object excluding the hands area. Also vibrotactile feedback and sound feedbacks are provided to the users according to the event.

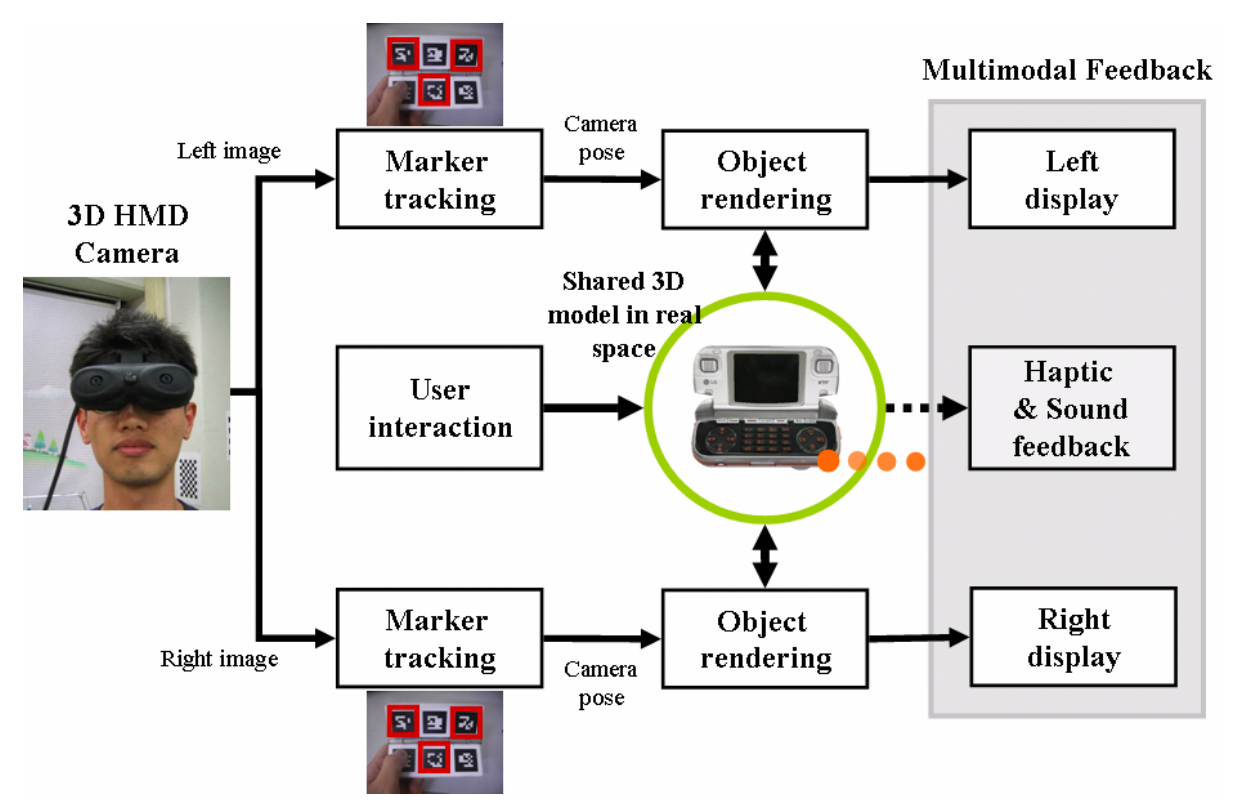

Fig. 1. Overall system

\subsection{Tangible Object Design}

Conventionally, users can not touch a virtual object because it has no physical presence or entity. However, by using tangible objects, users experience a touch sensation. In our work, diverse tangible objects and interaction methodologies are designed. Basically, multiple markers are attached to a tangible object so that the virtual object can be augmented when at least one marker is detected. It is necessary that several markers are hidden by hands when the user grasps the tangible object. The designed tangible object is a game-phone [6] which has a folder-type structure, as shown in Figure 2 (b) which is based on the CAD model in Figure 2 (a).

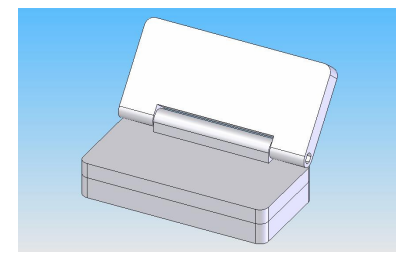

(a)

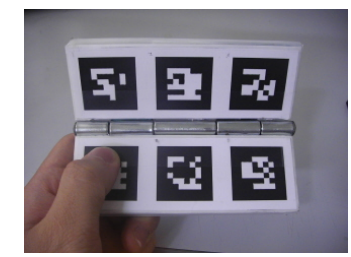

(b)

Fig. 2. (a) A CAD model (b) A folder-type Mock-up model 


\subsection{Reduction of the Hand Occlusion Problem}

In existing $\mathrm{AR}$ applications using the ARToolKit, augmented virtual objects can occlude with the user's hands because virtual objects just overlay the marker area as shown in Figure 6. This can cause visually awkward augmented images and also reduce immersion or naturalness [7]. Especially, the hands occlusion problem is critical when users interact with the augmented virtual objects by using their hands.

To reduce the hand occlusion problem [3], skin pixels are extracted through the adaptive threshold, but some of the parameters need to be manually set. Also, if the color balance is not correct, skin pixels can not be extracted properly. In [8], the authors assume that the marker's pattern information is already known and the size of the marker is greater than that of their hands. If the marker is small, the marker's pattern is complex, or the rotation angle is increased, then noise will also increase.

In order to compensate for the above problems, we propose the following method which enables hands segmentation under changing lighting conditions, with a relatively small marker area and in real-time.

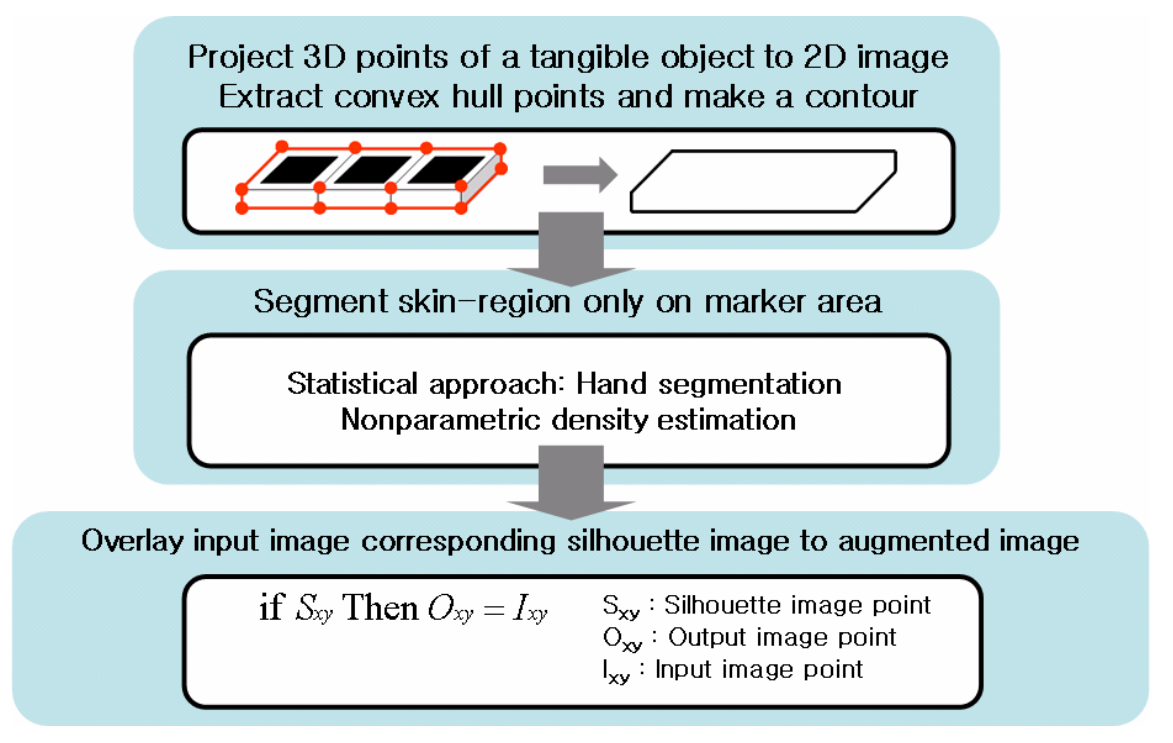

Fig. 3. Three steps for reducing the hands occlusion phenomenon

In the first step, by processing only the hands area on the tangible object, we can reduce the redundant time needed to process an entire image and thus improve processing speed. To do this, the $3 \mathrm{D}$ outer points of the tangible object are projected to a $2 \mathrm{D}$ image as shown in the upper part of Figure 3, and then the convex hull points are extracted by using the 3 coins algorithm [9]. From these points, contours (upper surface, bottom surface) are extracted and segment skin-regions only on this area.

In the second step, we apply the statistical approach to segment hand objects from the area of the tangible object. The hue and saturation components of the HSV color space are used to minimize the sensitivity of lighting. Through "Kernel Density 
Estimation" using "Parzen Windows"[10], we can estimate an unobservable underlying probability density function, based on observed data. In the histogram, the $\mathrm{x}$-axis is divided into bins of length $\mathrm{h}$. The probability of a sample $\mathrm{x}$ in a bin is estimated as follows.

$$
p \approx k_{N} / N
$$

Where $\mathrm{N}$ is the total number of samples, and $\mathrm{k}_{\mathrm{N}}$ is the number of samples in a bin. The corresponding probability is approximated by the frequency ratio. This approximation converges to the true $\mathrm{P}$ as $\mathrm{N} \rightarrow \infty$. The corresponding pdf value is assumed constant throughout the bin and is approximated by

$$
\hat{\mathrm{P}}(x) \equiv \hat{\mathrm{P}}(\hat{x}) \approx \frac{1}{h} \frac{k_{N}}{N}, \quad|x-\hat{x}| \leq \frac{h}{2}
$$

Where $\hat{x}$ is the midpoint of the bin and $\hat{p}(x)$ is the amplitude of the histogram curve. The $\mathrm{x}$-axis is divided into bins of length $\mathrm{h}$. Let $\mathrm{x}_{\mathrm{i}}, \mathrm{i}=1,2, \ldots, \mathrm{N}$, be the available feature vectors and define the function $\varphi(x)(4)$. The function is equal to 1 for all points inside the unit centered at the origin and 0 outside it. Then equation (2) can be rephrased as (3). We consider a window with the length of $\mathrm{h}$ centered at $\mathrm{x}$, the point where the pdf is to be estimated. The summation equals $\mathrm{k}_{\mathrm{N}}$. That is, the number of points falling inside this window. Then the pdf estimates results from dividing $\mathrm{k}_{\mathrm{N}}$ by $\mathrm{N}$ and the respective length $\mathrm{h}$.

$$
\begin{aligned}
& \hat{\mathrm{P}}(x)=\frac{1}{h}\left(\frac{1}{N} \sum_{i=1}^{N} \Phi\left(\frac{x_{i}-x}{h}\right)\right) \\
& \Phi\left(x_{i}\right)= \begin{cases}\frac{1}{\text { for }\left|x_{i}\right| \leq 1 / 2} \\
0 \quad \text { otherwise }\end{cases}
\end{aligned}
$$

The peaks of distributions should be detected. The first peak can be easily found in the histogram, being the bin with the largest value. The second peak is then detected by multiplying the histogram values by the square of the distance from the first peak [11]. This is the peak that is not close to the maximum. So, if the largest peak is at level $\mathrm{j}$ in the histogram, select the second peak as (6). The maximum value of the peaks is 256 . The valley between the distributions is calculated by equation 6 . From this data, threshold values are decided and segmentation is executed.

$$
\begin{aligned}
& \underset{0 \leq k \leq 255}{\arg \max }\left\{\left((k-j)^{2} \text { histogram }[k]\right)\right\} \\
& \text { if }(j>k) \quad \text { then } \underset{k \leq i \leq j}{\arg \min }\{\text { histogram }[i]\} \\
& \text { else } \underset{j \leq i \leq k}{\arg \min }\{\text { histogram }[i]\}
\end{aligned}
$$

In the last step, the extracted hand objects are overlaid again on the augmented virtual objects. In order to achieve this, a processor is added to the pipeline of existing ARToolKik. If pixels in the silhouette image exist, corresponding input pixels are overlaid on the augmented virtual object again. As a result, users interacting with 
augmented virtual objects by hand will experience better immersion and more natural images.

$$
\begin{aligned}
& \text { If } S_{x y} \text { exists } \\
& \text { then } A_{x y}=R_{x y}
\end{aligned}
$$

Where $S_{x y}$ is the silhouette image coordinates, $A_{x y}$ is the augmented image coordinates and $\mathrm{R}_{\mathrm{xy}}$ is the input image coordinates respectively.

\subsection{Multimodal Feedback Focused on the Vibrotactile Feedback}

In order to provide immersive interaction, multisensory feedback combining visual and auditory effects is used. However, the importance of haptic feedback is increased in manipulation and exploration tasks. David Feygin, et al. explained the role of haptics in training [12] and Miriam Reiner emphasised the need of haptic feedback to provide better realism [13].

There have been some attempts to combine tactile feedback into augmented reality. Working with urban planning workspaces, V. Buchmann, et al. used buzzers mounted on gloves so that users were able to feel the vibration when their hand was in contact with AR models of city blocks [14]. Thus they could provide immersive interactions to users.

In our game application scenario (e.g. the racing game), if a player collides with a wall, a vibration is generated and an explosive sound is played. A vibrotactile module is inserted into the tangible object as shown in Figure 4. A wireless communication module (Bluetooth:ADCODE-300) for a vibrotactile module was used. Pancake style vibrators were selected due to their similarity to real vibrating motors in real cellular phones. The vibration module can be controlled at seven levels. 0 is the lowest level and 6 is the highest level of intensity. If the car is on the road, then the 2 3 levles is applied, but if the car collides with a wall or other cars, the 6 step of intensity is applied.

Therefore sight, touch and auditory senses are provided to the users simultaneously, resulting in better immersion as the users interact with virtual objects in the augmented reality.

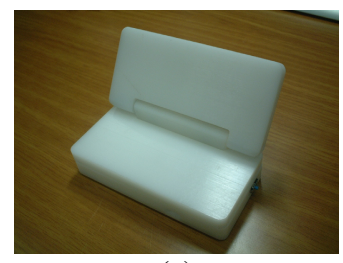

(a)

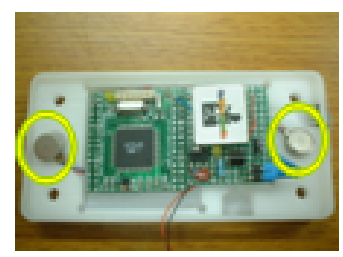

(b)

Fig. 4. (a) A tangible object (b) Inner part of tangible object

\section{Implementation and Experiment Result}

\subsection{Experiment Environment}

In our system, we used a 3D video see-through HMD of VRmagic corporation [15]. Cameras were attached on the positions of each eye. The frame rate was 30 (f/s) and 
resolution was $640 * 480$ pixels. The two input images from cameras were processed in Dell Corp.'s workstation (e.g. 650MT). An output image was resized to $800 * 600$ to display on LCD in HMD. We referenced the OpenCV version 5.0 beta [16] and ARToolKit version 2.70 beta [17] libraries. The experiment environment was an indoor complex background. As shown in Figure 5, the user wearing the HMD interacts with the virtual object by grasping a tangible object. This tangible object is a mock-up model of a game-phone to which fiducial markers are attached. The user sees a virtual game-phone through the HMD.

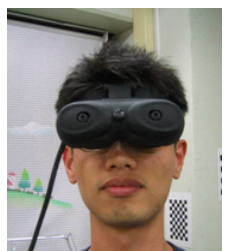

(a)

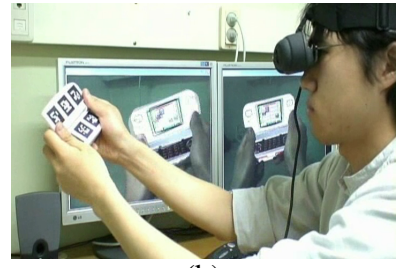

(b)

Fig. 5. (a) the user wearing the HMD (b) the user can interact with a tangible object

\subsection{Hands Object Segmentation}

Figure 6 (a-c) represent input images and Figure 6 (a'-c') show contours (upper surface, bottom surface). Processing only the hands area on this area, reduces the redundant time needed to process an entire image.

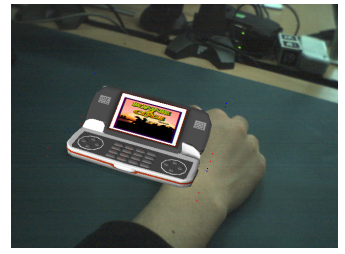

(a)

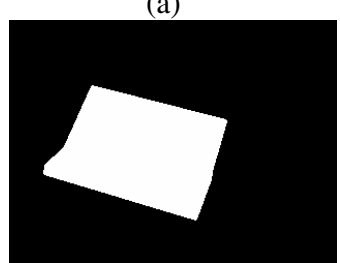

(a')

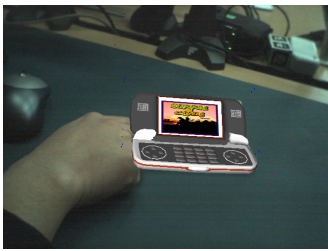

(b)

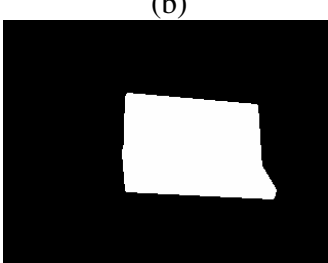

(b’)

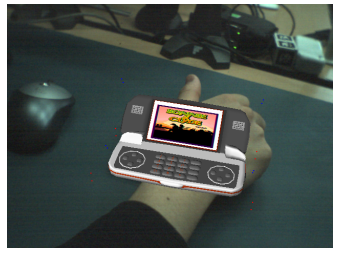

(c)

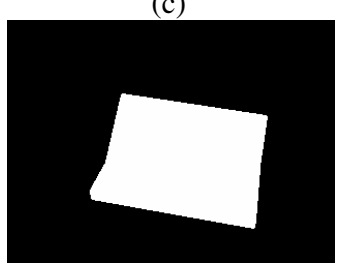

(c')

Fig. 6. (a-c) Hand occluded images (a'-b') Extract convexes (the upper side and bottom side of a convex)

Through "Kernel Density Estimation" using "Parzen Windows", we can estimate unobservable underlying probability density function, based on observed data. Figure 7 (a-c) shows the histograms of the pixels in hands objects corresponding with the images of Figure 6 ( $\left.a^{\prime}-c^{\prime}\right)$. We can see the variances of distributions according to the 
area of hands objects. Figure 7 (a'-c') shows the results of "Kernel Density Estimation".

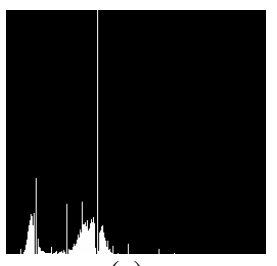

(a)

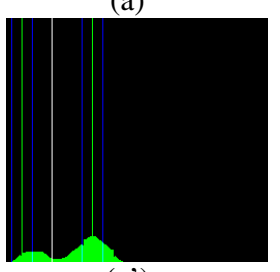

(a')

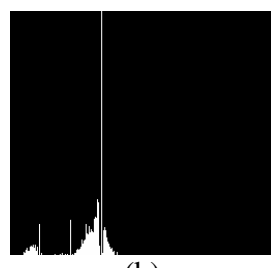

(b)

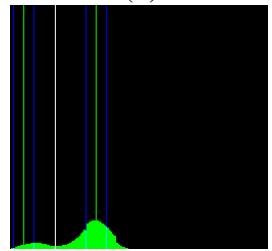

(b')

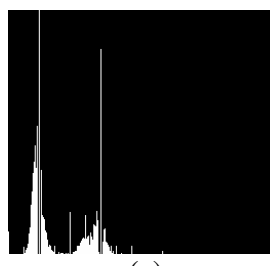

(c)

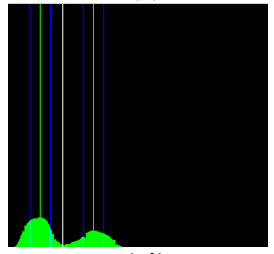

(c')

Fig. 7. (a-c) Histograms corresponding the Figure 6. (a'-c') Results of "Kernel Density Estimation".

Our method partially solves the hand occlusion problem, providing better immersive and more natural images to the users when they interact with virtual objects by hands. Also hands object segmentation under various lighting condition is possible, as shown in Figure 8 (a'-c').
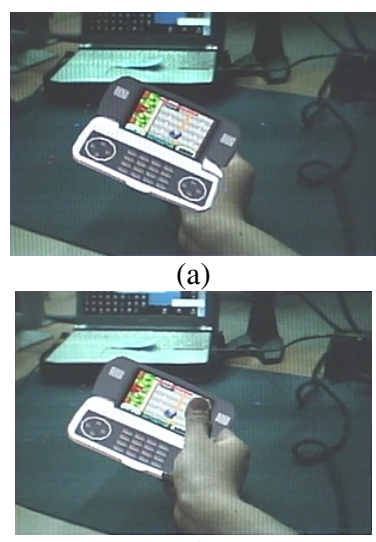

(a')

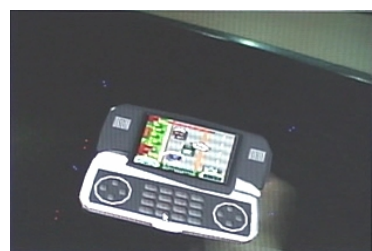

(b)

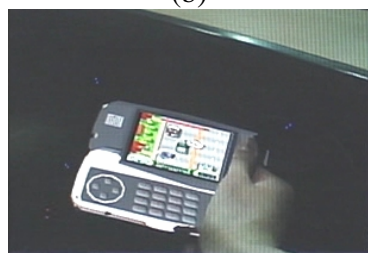

(b')

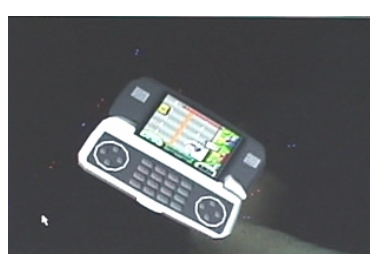

(c)

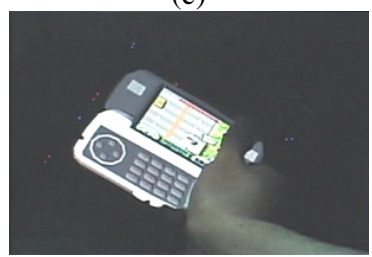

(c')

Fig. 8. Hands object segmentation under various lighting conditions

\subsection{Game Scenario}

Figure 9 shows the racing game in action. The users can obtain multimodal feedback with a virtual game-phone using the tangible object. The users can control the car by tilting the tangible object in the direction of $\mathrm{x}, \mathrm{z}$ axis. If a player collides with a wall, vibration feedback is generated and an explosive sound is played. 


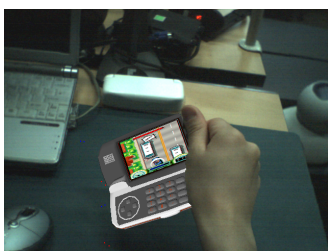

(a)

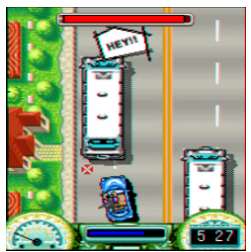

(b)

Fig. 9. Game control by tilting the tangible object

\section{Conclusion and Future Work}

In this paper, we proposed methodologies for better immersion and interaction in ARbased product design. By adapting the tangible object touch sensations can provide to the users. Also we have partial solved the hand occlusion problem, providing more immersive or natural images to the users. We improve user interaction with tangible objects by multimodal feedback, focusing on vibrotactile feedback.

Using the proposed methodologies, we realized our immersive model in a gamephone, where users control cars in a racing game by tilting a tangible object. The proposed methodologies contribute to the immersive realization of the conventional AR system.

In future work, we will add other cues for more robust hands segmentation such as edge and geometry relations of hands models. Also we are considering interaction with virtual objects using fingertips by exploiting 3D depth information and the augmentation by exploiting points tracking of a tangible object, even though all of markers are not detected.

\section{References}

[1] Klinker, G., Dutoit, A.H. Bauer, M. Bayer, J. Novak, V. Matzke, D, "Fata Morgana - a presentation system for product design", ISMAR 02, 30 Sept.-1 Oct. 2002, pp. 76 - 85

[2] Fiorentino, M. de Amicis, R. Monno, G. Stork, A., "Spacedesign : A Mixed Reality Workspace for Aesthetic Industrial Design”, ISMAR 02, 30 Sept.-1 Oct. 2002, pp. 86 318

[3] Woonhun Lee, Jun Park "Augmented Foam: A Tangible Augmented Reality for Product Design”, Woohun Lee, Jun Park, ISMAR 05, 5-8 Oct. 2005, pp. 106 - 109

[4] Phantom device, http://www.sensable.com/products/phantom_ghost/phantom.asp

[5] "An introduction to kernel density estimation", http://www.maths.uwa.edu.au/ duongt/seminars/intro2kde/

[6] LG-KV 3600, http://www.cyon.co.kr/good/product/product_view1.jsp?product_id=165

[7] Gun A. Lee, Claudia Nelles, Mark Billinghurst, Gerard Jounghyun Kim, "Immersive Authoring of Tangible Augmented Reality Application”, ISMAR 04, 2-5 Nov. 2004 Page(s): 172 - 181

[8] Shahzad Malik, Chris McDonald, Gerhard Roth, "Hand Tracking for Interactive Patternbased Augmented Reality", ISMAR 02, 30 Sept.-1 Oct. 2002 Page(s):117 - 126

[9] Avis, D. and Toussaint. An optimal algorithm for determining the visibility of a polygon from an edge. IEEE Trans. Comput. 30, 910-914 
[10] Sergios theodoridis and konstantinos koutroumbas, "Classifiers based on Bayes decision theory", Book of Pattern recognition, Elsevier academic press

[11] J. R. Parker, "Advanced methods in grey-level segmentation", algorithms for image processing and computer vision, Wiley

[12] David Feygin, Madeleine Keehner, Frank Tendick, "Haptic Guidance: Experimental Evaluation of a Haptic Training Method for a Perceptual Motor Skill", Proceedings of 10th International Symposium in Haptic Interfaces for Virtual Environment and Teleoperator Systems (Haptics 2002), Orlando, FL, pp. 40-47, March, 2002.

[13] Miriam Reiner, "The Role of Haptics in Immersive Telecommunication Environments", IEEE TRANSACTIONS ON CIRCUITS AND SYSTEMS FOR VIDEO TECHNOLOGY, VOL. 14, NO. 3, MARCH, 2004.

[14] Buchmann, S. Violich, M. Billinghurst, "A. Cockburn. FingARtips: gesture based direct manipulation in Augmented Reality", In Proceedings of the 2nd international conference on Computer graphics and interactive techniques in Australasia and SouthEast Asia (Graphite 2004). 15-18th June, Singapore, 2004, ACM Press, New York, New York, pp. 212-221.

[15] VRmagic, http://www.vrmagic.com/index_e.html

[16] Intel OpenCV Library, http://www.intel.com/research/mrl/research/opencv/

[17] ARToolKit, http://www.hitl.washington.edu/ARToolKit 